\title{
A influência de exercícios na infância e ganhos na saúde para o futuro
}

\author{
The influence of childhood exercise and health gains for the future \\ La influencia del ejercicio infantil y los beneficios para la salud en el futuro
}

Gabriel de Sousa Carneiro ORCID: https://orcid.org/0000-0002-0881-7870 Faculdade Maurício de Nassau, Brasil E-mail: gabrieldesousa2735@gmail.com

Nágila Iane Pacheco ORCID: https://orcid.org/0000-0002-2836-1639 Centro Universitário Unifacid/Wyden, Brasil Email: nagilaiane@hotmail.com

Jorge Davi de Sousa e Silva ORCID: https://orcid.org/0000-0003-3157-1108

Centro Universitário UniFacid/Wyden, Brasil E-mail: jddavisilva123@gmail.com

Lorran André Moraes

ORCID: https://orcid.org/0000-0002-3858-3059 Universidade Federal do Piaú, Brasil

E-mail: lorranbio@hotmail.com

Edilma Mendes Rodrigues Gonçalves ORCID: https://orcid.org/0000-0003-3727-5430 Universidade Federal do Piauí, Brasil E-mail: edilmamendes@cte.uespi.br

Danielle Costa Lopes

ORCID: https://orcid.org/0000-0002-7382-1323 Universidade Federal do Piauí, Brasil

E-mail: dany197_@hotmail.com

Alciene Pacheco da Silva

ORCID: https://orcid.org/0000-0003-4117-2792 Universidade Federal do Piauí, Brasil

E-mail: alcienepacheco@ufpi.edu.br

Adryana Ryta Ribeiro Sousa Lira ORCID: https://orcid.org/0000-0002-1639-3369 Centro Universitário UniFacid/Wyden, Brasil

E-mail: dryribeiro22@gmail.com

Diego Rodrigues Pessoa

ORCID: https://orcid.org/0000-0002-8981-673X Universidade Federal do Piaú, Brasil

E-mail: fisio.diegorodrigues@gmail.com

Leandro Ferreira Frade Soares

ORCID: https://orcid.org/0000-0003-1499-431X

Universidade Federal de Pernambuco, Brasil

E-mail: leandro_frade30@hotmail.com

Guilherme Reis Nunes Camarço

ORCID: https://orcid.org/0000-0002-9450-6314

Centro Universitário UniFacid/Wyden, Brasil

E-mail: guilherme_reis76@hotmail.com

Ivanira Vieira Loiola Coutinho

ORCID: https://orcid.org/0000-0003-1877-1885

Centro Universitário Unifacid/Wyden, Brasil E-mail: ivanirafarma@gmail.com

Ian Carlos Cavalcante Vasconcelos

ORCID: https://orcid.org/0000-0002-7642-7284 Faculdade Maurício de Nassau, Brasil E-mail:ianvasconcelos10@gmail.com

Bruna Ellen Ribeiro de Oliveira ORCID: https://orcid.org/0000-0002-5728-5196 Faculdade Maurício de Nassau, Brasil E-mail: brunasr588@gmail.com

Lyslly Rhanny Soares De Deus

ORCID: https://orcid.org/0000-0003-4367-5609

Centro Universitário Unifacid/Wyden, Brasil E-mail: lysllyrhanny@hotmail.com 


\title{
Luiza Aragão Paiva Pires Ferreira Mendes ORCID: https://orcid.org/0000-0002-4017-6102 Centro Universitário Unifacid/Wyden, Brasil E-mail: luizamendes0412@gmail.com
}

\begin{abstract}
Resumo
Introdução: A infância é uma das partes mais significativas para sua saúde da criança, pois no decorrer deste período/fase acontecem processos essenciais no crescimento e desenvolvimento. Na direção de auxiliar o apoio a população para atingir as metas e manter um cotidiano saudável, todos os países são notificados pela World Health Assembly (WHA) a desenvolver uma execução política de programas nacionais e subnacionais, no intuito de autorizar que pessoas de idades e com capacidades diversas, sejam fisicamente ativas e saudáveis. Assim, esse estudo teve como objetivo abordar e relatar os benefícios da atividade física desenvolvida na infância e na vida adulta, a importância de uma vida ativa, as pressuposições e os riscos de possuir uma vida sedentária. Metodologia: trata-se de uma pesquisa de natureza exploratória, bibliográfica utilizando artigos científicos disponíveis nas bases de periódicos, como: BVS, Fiocruz, Faculdade AGES, Google School, Instituto IDEIA, IFC, LILACS, Multidisciplinary Reviews, PUC Goiás, PubMed, Revista Científica Multidisciplinar Núcleo do Conhecimento, REASE, SCIELO, UPF, UFSM, USP, WHO. Resultado e Discussões: Independente do período em que se encontra, a atividade física consegue apresentar inúmeros benefícios para o praticador, como exemplo, aperfeiçoando o crescimento das crianças, influenciando na obtenção de uma boa qualidade de vida e saúde. A atividade física é um processo que conjuntamente com o ambiente, a nutrição e a genética, auxiliam para que o indivíduo alcance seu potencial de crescimento e desenvolvimento, que desenvolva, absolutamente, a capacidade física e tenha em concordância no resultante um favorável nível de saúde. Conclusão: A atividade física é indicada para qualquer faixa etária em um processo que atua diretamente no desenvolvimento motor da criança, no combate ao sedentarismo, o que as torna mais ativas, desde que seja realizada com acompanhamento de um profissional habilitado (Educador físico).
\end{abstract}

Palavra-chave: Atividade física; Exercício físico; Criança; Benefícios.

\begin{abstract}
Introduction: Childhood is one of the most significant parts of your child's health, as essential processes in growth and development take place during this period/phase. In order to help support the population to achieve the goals and maintain a healthy daily life, all countries are notified by the World Health Assembly (WHA) to develop a policy implementation of national and subnational programs, in order to authorize people of ages and with diverse abilities, are physically active and healthy. Thus, this study aimed to address and report the benefits of physical activity developed in childhood and adulthood, the importance of an active life, the assumptions and risks of having a sedentary life. Methodology: this is an exploratory, bibliographic research using scientific articles available in the databases of journals, such as: BVS, Fiocruz, Faculdade AGES, Google School, Instituto IDEIA, IFC, LILACS, Multidisciplinary Reviews, PUC Goiás, PubMed, Revista Multidisciplinary Science Center for Knowledge, REASE, SCIELO, UPF, UFSM, USP, WHO. Results and Discussions: Regardless of the period in which it is, physical activity can present numerous benefits for the practitioner, for example, improving the growth of children, influencing the achievement of a good quality of life and health. Physical activity is a process that, together with the environment, nutrition and genetics, helps the individual to reach his/her potential for growth and development, to develop, absolutely, physical capacity and have, in the result, a favorable level of health. Conclusion: Physical activity is indicated for any age group in a process that acts directly on the child's motor development, in the fight against sedentary lifestyle, which makes them more active, as long as it is carried out with the accompaniment of a qualified professional (Physical Educator).
\end{abstract}

Keywords: Physicalactivity; Physicalexercise; Childhood; Benefits.

\section{Resumen}

Introducción: La infancia es una de las partes más significativas de la salud de su hijo, ya que durante este período/fase tienen lugar procesos esenciales de crecimiento y desarrollo. Con el fin de ayudar a apoyar a la población a alcanzar las metas y mantener una vida diaria saludable, la Asamblea Mundial de la Salud (WHA) notifica a todos los países para que desarrollen una política de implementación de programas nacionales y subnacionales, con el fin de autorizar a las personas de edades y con habilidades diversas, son físicamente activos y saludables. Así, este estudio tuvo como objetivo abordar y relatar los beneficios de la actividad física desarrollada en la infancia y la edad adulta, la importancia de una vida activa, los supuestos y riesgos de tener una vida sedentaria. Metodología: se trata de una investigación bibliográfica, exploratoria, utilizando artículos científicos disponibles en las bases de datos de revistas como: BVS, Fiocruz, Faculdade AGES, Google School, Instituto IDEIA, IFC, LILACS, Revisiones Multidisciplinares, PUC Goiás, PubMed, Revista Multidisciplinar Ciencia Centro del Conocimiento, REASE, SCIELO, UPF, UFSM, USP, OMS. Resultados y Discusiones: Independientemente del período en que se encuentre, la actividad física puede presentar numerosos beneficios para el practicante, por ejemplo, mejorando el crecimiento de los niños, influyendo en el logro de una buena calidad de vida y salud. La actividad física es un proceso que, junto con el medio ambiente, la nutrición y la genética, ayuda al individuo a alcanzar su potencial de crecimiento y desarrollo, a 
desarrollar, en forma absoluta, la capacidad física y tener, en consecuencia, un nivel de salud favorable. Conclusión: La actividad física está indicada para cualquier grupo de edad en un proceso que actúa directamente en el desarrollo motor del niño, en la lucha contra el sedentarismo, lo que lo vuelve más activo, siempre que se realice con el acompañamiento de un profesional calificado (Educador Físico).

Palabras clave: Actividad física; Ejercicio físico; Infancia; Beneficios.

\section{Introdução}

Em 2018, a World Health Assembly (WHA) aprovou um Plano de Ação Mundial sobre movimento Físico (GAPPA) de 2018-2030 (Who, 2018), o qual, considerou uma recente meta global voluntária para sintetizar os níveis de inatividade física em adultos e jovens em 15\% até 2030. É importante destacar que, como parte da deliberação da WHA, os países Membros solicitaram que a Organização Mundial de Saúde (OMS) renovasse as Diretrizes Globais de 2010 sobre o movimento físico para o bem-estar populacional (Bull et al., 2020).

Vale ressaltar que, as diretrizes nacionais e mundiais sobre atividade física (AF) é um elemento central de um governo que se preocupa e estabelece uma base política para ações de saúde pública. Nesse sentido, a OMS solicita que todos os países estabeleçam diretrizes nacionais e definam seus objetivos de acordo com cada realidade territorial. Para os autores, os países são notificados e estimulados a desenvolver uma execução política de programas nacionais e subnacionais, no intuito de autorizar que as pessoas de idades e capacidades diversas, sejam fisicamente ativas e saudáveis, contribuindo assim, na direção de auxiliar os mesmos para atingir as metas e manter um cotidiano saudável (Bull et al., 2020).

Hoje, sabe-se que a infância é uma das etapas mais significativas para a saúde da criança, logo no decorrer deste período acontecem processos essenciais no crescimento e desenvolvimento. Neste período da vida, desenvolve a parte motora, no qual é reconhecida como um processo sequencial e contínuo. Além disso, é nesse momento que a criança adquire um elevado número de habilidades e competências, evoluindo, por exemplo, os movimentos desorganizados e simples para complexos e sistemáticos (Xavier, 2018).

O desenvolvimento motor integrado é o processo de mudança no comportamento, movimento e postura de um ser ao longo da vida. Destaca-se ainda, que este processo sofre influência direta do conjunto de características genéticas, relacionadas à predisposição para o movimento e as experiências de cada, assim, esses movimentos originam-se decorrente de demandas intrínsecas (i.e. próprias de cada indivíduo), extrínsecas (i.e. externas, do ambiente) e depende de interações complexas entre diferentes componentes e sistemas do corpo humano, principalmente neurofisiológicos e psicológicos (Gallahue \& Ozmun, 2003; Cech \& Martin, 2011).

Uma das vertentes do desenvolvimento motor integrado é o homeostasia, ou seja, a capacidade física de adaptar o indivíduo ao ambiente, em estado de repouso (equilíbrio quase estático) ou de mobilidade, quando sujeitado a estímulos diversos (equilíbrio dinâmico), possibilitando orientação e estabilidade. Nesse sentido, para que a pessoa exerça um controle postural correto é necessária uma interação harmônica entre o sistema nervoso mediante a interação entre os sistemas neurais proprioceptivo, vestibular, musculoesquelético e visual, os quais entendem as relações biomecânicas e neurofuncionais (Nunes et al., 2019).

Outra informação importante a ser destacada é quanto à obesidade, pois essa é um problema global, reconhecido por ser um fator de risco comum de várias outras doenças crônicas. Estudos demonstram que, a intensificação da globalização e a industrialização de produtos alimentícios têm gerado profundas consequências a saúde humana, com isso a mudança no estilo de vida passou por pontos negativos, incluindo substituições nos hábitos alimentares e na redução do exercício físico (EF), podendo contribuir em parte para a obesidade (Silva et al., 2021).

Em consonância, analises preliminar revelam que é na fase infantil a melhor época para prevenção da obesidade. Contudo, é possível conseguir controlar melhor esse fator na vida adulta, porque está relacionado a mudanças nos hábitos e 
disponibilidade dos pais enquanto eram crianças. Dessa forma, é relevante a estimulação desde cedo nas crianças acerca do costume, o hábito da prática de Educação Física $(E F)$ e do uso de uma alimentação saudável equilibrada, pois se sabe que ambos são os principais meios para a criação de um estilo de vida saudável para os jovens. Com isso, recomenda-se para essa faixa etária, evitar o alto consumo de carboidrato, lipídio, gordura e, ampliar o consumo de frutas e vegetais correlacionandoos sempre que possível com exercícios e atividades físicas (Silva et al., 2021).

Desta forma, este estudo têm como objetivo abordar acerca do período da infância das crianças e relatar os benefícios da atividade física desenvolvida na infância e juventude, além também da importância de uma vivência ativa, as pressuposições e os riscos do sedentarismo. Ademais, busca-se expor a respeito dos benefícios e precauções que precisam ser comunicados pelos pais de maneiras constantes a criança referente a prática da EF.

\section{Metodologia}

A pesquisa realizada é de natureza exploratória, do tipo bibliográfica (Iucatan, 2020), sobre "A influência de exercícios na infância e ganhos na saúde para o futuro", através da consulta em bancos de dados online para o levantamento das informações. As principais bases de dados de periódicos utilizados para as buscas ativas dos artigos foram: Biblioteca Virtual em Saúde (BVS), Fundação Oswaldo Cruz (Fiocruz), Faculdade AGES, Google School, Instituto IDEIA, Instituto Federal Catarinense (IFC), Literatura Latino-americana e do Caribe em Ciências da Saúde (LILACS), Multidisciplinary Reviews, PUC Goiás, PubMed, Revista Científica Multidisciplinar Núcleo do Conhecimento, Revista Ibero-Americana de Humanidades, Ciências e Educação (REASE), Scientific Electronic Library Online (SCIELO), Universidade de Passo Fundo (UPF), Universidade Federal de Santa Maria (UFSM), Universidade de São Paulo (USP), World Health Organization (WHO).

Os critérios de inclusão sobre o referido levantamento deram-se através do uso das combinações de palavras-chaves em inglês "Physical activity in childhood, physical education for children, childhood health, physical activityand benefits", e as palavras em português “Atividade física na infância, educação física infantil, saúde infantil, atividade física e benefícios”. Com o intuito de tornar a busca mais específica, realizou-se as conexões entre os termos com a utilização dos operadores booleanos "AND", que na íntegra retrata-se da temática referente à atividade física na infância para a saúde. Como critérios de exclusão, eliminaram-se 54 artigos de 76 encontrados, limitando-se os resultados das buscas em inglês, espanhol e português, em seguida das compilações resolveu-se as divergências por consenso em relação à duplicidade, as não relacionadas à temática e fora do período de tempo. Neste estudo, as referências bibliográficas selecionadas e utilizadas na pesquisa foram publicadas e indexadas nos referidos bancos de dados dos anos de 2018 a 2021.

Dessa forma, nesse levantamento são reportados 47 artigos da BVS, 1 da Fiocruz, 1 da Faculdade AGES, 2 da Google School, 1 da Instituto IDEIA, 1 do IFC, 1 LILACS, 1 da Multidisciplinary Reviews, 1 da PUC Goiás, 3 da PubMed, 1 da Revista Científica Multidisciplinar Núcleo do Conhecimento, 1 da REASE, 15 da SCIELO, 1 da UPF, 1 da UFSM, 1 da USP e 1 da WHO como demonstra-se no quadro a seguir (Figura 1).

Todas as pesquisas, como a triagem de títulos, resumos e a seleção de estudos foram executadas pelos pesquisadores que trabalharam de forma independente. Em seguida das compilações, resolveu-se as divergências por consenso em relação a duplicidade e as não relacionadas a temática. Assim, utilizaram-se todos os artigos considerados potencialmente legíveis para revisão do seu texto completo. Após isso, para a análise dos dados foi utilizado o programa Microsoft Excel versão Windows 10. 
Figura 1: Fluxograma com as etapas de análise realizadas para seleção dos artigos científicos.

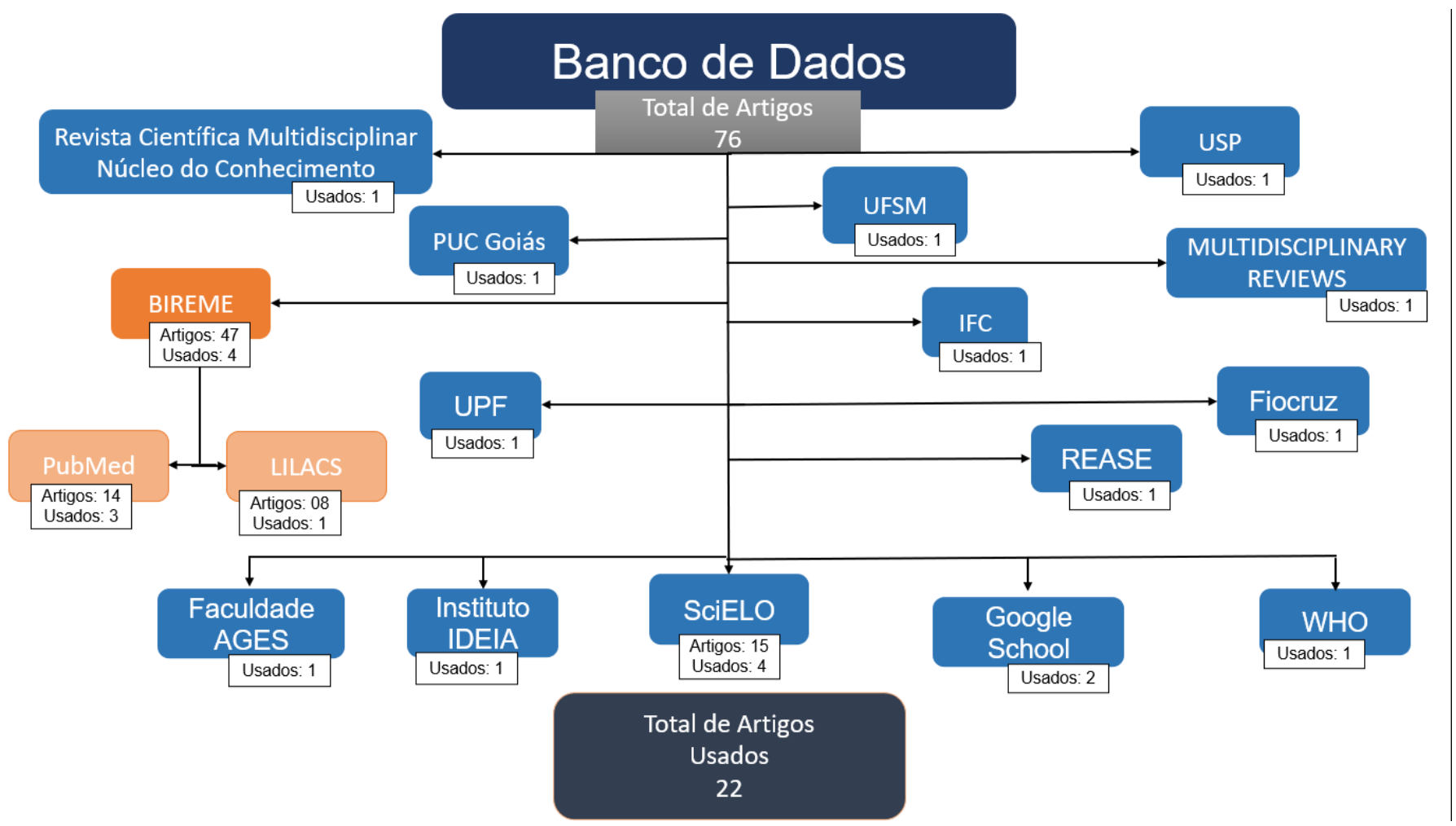

Fonte: Sousa (2021).

O Quadro 1 apresenta a listagem das principais publicações encontradas na busca ativa nos principais bancos de dados de periódicos, acerca da temática "A influência de exercícios na infância e ganhos na saúde para o futuro". 
Quadro 1: Artigos científicos listados dos bancos de dados

\begin{tabular}{|c|c|c|}
\hline Título do artigo & $\begin{array}{l}\text { Autores e ano da } \\
\text { pesquisa }\end{array}$ & $\begin{array}{l}\text { Revista científica } \\
\text { /Periódico }\end{array}$ \\
\hline Compreendendo o Desenvolvimento Motor: bebês, crianças, adolescentes e adultos. & (Gallahue, 2003) & Google School \\
\hline Motor Development. Functional Movement Development Across the Life Span-E-Book. & $($ Cech, 2011) & Google School \\
\hline $\begin{array}{l}\text { Importância da Atividade Física na Infância. Revista Científica Multidisciplinar Núcleo } \\
\text { do Conhecimento }\end{array}$ & (Arraz, 2018) & $\begin{array}{c}\text { Revista Científica } \\
\text { Multidisciplinar Núcleo do } \\
\text { Conhecimento }\end{array}$ \\
\hline $\begin{array}{l}\text { Worldwide trends in insufficient physical activity from } 2001 \text { to } 2016 \text { : a pooled analysis } \\
\text { of } 358 \text { population-based surveys with } 1.9 \text { million participants. }\end{array}$ & (Guthold, 2018) & PubMed \\
\hline $\begin{array}{l}\text { Educação Física Escolar na Infância e Adolescência e sua influência na adoção da } \\
\text { Atividade Física na vida adulta. }\end{array}$ & (Oliveira, 2018) & IFC \\
\hline A importância do desenvolvimento motor na primeira infância. & (Xavier, 2018) & Fiocruz \\
\hline $\begin{array}{l}\text { Plano de ação global para atividade física 2018-2030: pessoas mais ativas por um } \\
\text { mundo mais saudável. }\end{array}$ & (Who, 2018) & WHO \\
\hline $\begin{array}{l}\text { Conhecimento de adolescentes acerca dos benefícios do exercício físico para a saúde } \\
\text { mental. }\end{array}$ & (Campos, 2019) & Scielo \\
\hline A influência da atividade física no aprendizado com idade escolar. & (Freitas, 2019) & $\begin{array}{l}\text { Revista Científica Do } \\
\text { Instituto Ideia }\end{array}$ \\
\hline $\begin{array}{l}\text { Associação entre práticas de atividade física e desempenho acadêmico de estudantes } \\
\text { chilenos do ensino fundamental e médio. }\end{array}$ & (Lima, 2019) & Scielo \\
\hline Controle postural na infância: efeitos do Método Pilates sobre o equilíbrio. & (Nunes, 2019) & LILACS \\
\hline $\begin{array}{l}\text { World Health Organization } 2020 \text { guidelines on physical activity and sedentary } \\
\text { behaviour }\end{array}$ & (Bull, 2020) & PubMed \\
\hline $\begin{array}{l}\text { Padrões de comportamentos de risco e de proteção para doenças crônicas não } \\
\text { transmissíveis na população adulta e infantil do Brasil. }\end{array}$ & (Carvalho, 2020) & USP \\
\hline Alimentação saudável: educando e cuidando da infância na educação infantil. & (Giaretta, 2020) & UPF \\
\hline $\begin{array}{l}\text { Global trends in insufficient physical activity among adolescents: a pooled analysis of } \\
298 \text { population-based surveys with } 1.6 \text { million participants }\end{array}$ & (Guthold, 2020) & PubMed \\
\hline A contribuição do treinamento funcional para a diminuição da obesidade infantil. & (Iucatan, 2020) & PUC Goiás \\
\hline $\begin{array}{l}\text { Self-reported level of physical activity in schoolchildren who participate in physical } \\
\text { education: a systematic review }\end{array}$ & (López-gil, 2020) & Scielo \\
\hline $\begin{array}{l}\text { Educação Física e Saúde em Tempos de Covid-19. Observatório Socioeconômico da } \\
\text { COVID-19. }\end{array}$ & (Daronco, 2021) & UFSM \\
\hline $\begin{array}{l}\text { Doenças crônicas não transmissíveis e mudanças nos estilos de vida durante a pandemia } \\
\text { de COVID-19 no Brasil. }\end{array}$ & (Malta, 2021) & Scielo \\
\hline $\begin{array}{l}\text { Atividade física como prevenção da obesidade e síndrome metabólica na infância e } \\
\text { adolescência: uma revisão integrativa. }\end{array}$ & (Nunes, 2021) & Multidisciplinary Reviews \\
\hline $\begin{array}{l}\text { Benefícios das práticas de atividades físicas em crianças no âmbito escolar. Artigo de } \\
\text { conclusão de curso }\end{array}$ & (Sátiro, 2021) & Faculdade Ages \\
\hline A importância da educação física na vida de crianças obesas & (Silva, 2021) & REASE \\
\hline
\end{tabular}

Fonte: Sousa (2021). 


\section{Resultados e Discussão}

\subsection{Abordagens da EF da infância a velhice}

Através do contexto didático é permitido dividir o espaço de vivência das pessoas em fases, na qual cada uma com particularidades e transformações complexas de comprovação (Oliveira, 2018). Nessas etapas, a infância e a juventude são os ciclos primários, seguindo pela fase adulta e velhice. Nessa perspectiva Oliveira (2018), alega que o progresso do sujeito não ocorre de maneira padrão para todos, a maturação compreende mudanças expressivas entre os humanos. Ainda para os autores, essa maturidade é correlacionada as variações sociais dos últimos tempos os quais induziram em alterações nos períodos da vida, sobretudo, nas primeiras, desde o nascimento até a puberdade, ocorrendo uma época de renovações, com a capacidade de acarretar algumas complicações comportamentais, agregados ao modelo de vida e questões socioculturais.

Cabe destacar que a infância é um estágio nativo, continuamente apontado pela mobilidade corporal, ao lado de atividades ao ar livre (Iucatan, 2020). Segundo as continuas alterações que intercorrem nessa fase, é pertinente destacar a relevância na estruturação de valores consideráveis em todos os momentos da vivência. O sedentarismo, por exemplo, apresenta um amplo acréscimo, voltados as atividades diárias que consistem em jogos no computador, assistir televisão e nos demais hábitos danosos à saúde. Ademais, potencializa a demanda de permanecer ativo e apresenta como benefício fisiológico, o melhoramento do ânimo, atenuação do estresse e adição da habilidade cognitiva (Carvalho, 2020).

Pressupondo que a capacidade da criança em coordenar os sistemas de controle postural aumenta com a prática cotidiana, tornando-se proporcional pela sua tentativa de experimentar com a sua habilidade em coordenar os sistemas neurais responsáveis pelo equilíbrio. Desse modo, uma vida ativa desempenha uma importante influência no padrão de desenvolvimento motor e no crescimento da pessoa. No entanto, na sua privação pode promover impacto negativo no âmbito postural (Nunes et al., 2019). Independentemente da promoção de saúde abranger variados fatores, há indícios que o hábito regular de exercícios desde o período infantil, contém grandes possibilidades de permanecerem na pessoa e futuramente possibilitar uma vida ativa e saudável (Iucatan, 2020).

Logo, a adolescência que vai dos 10 a 19 anos, é conceituada por apresentar na pessoa várias etapas de transformação estrutural e comportamental, principalmente nos aspectos psicossociais, físico e biológico. De acordo com as diretrizes da OMS, essa etapa da vida é dividida em três estágios: pré-adolescência (10-13 anos), meia-adolescência (14-16 anos) e final da adolescência (17-19 anos) (Campos et al., 2019).

Durante essa fase, o cérebro passa por mudanças substanciais devido ao desenvolvimento e aperfeiçoamento próprio e habitual do sujeito. Essas são modificações necessárias para permitir que desenvolvam e alcancem, posteriormente, um cérebro equilibrado, autônomo e saudável. Ao mesmo tempo, compromete uma preocupação para as doenças mentais que conseguem manifestar-se (Campos et al., 2019). Já na juventude é possível perceber geralmente, um grupo de alterações emocionais, sociais, cognitivas e biológicas, notada pela auto-suficiência e emancipação, excetuando-se uma nova conduta (Iucatan, 2020).

Oliveira (2018) e López et al., (2020), ressaltam que em sua tarefa diária os jovens precisariam efetuar em geral uma hora ou mais de exercício, entre cinco dias ou mais na semana, pois sabe-se que a EF corrobora, especialmente, no aprimoramento da disposição física, na prevenção das Doenças Crônicas Não Transmissíveis (DCNT), no acrescentamento da densidade óssea e no melhoramento do bem-estar. De acordo com os autores, isso se faz importante, visto que, aperfeiçoa o perfil antropométrico e bioquímico, reduz as taxas de enfermidades referentes à obesidade, apontando uma volumosa eficácia na diminuição do índice de massa corporal (IMC) e do nível basal de pressão arterial sistólica e diastólica.

É notável salientar a aparição da obesidade, que atualmente desenvolvem-se, gradativamente, para o público jovem, em especial pelos atuais hábitos da vida moderna. Nesse caso, ressalta-se que é uma doença que atingir todas as faixas etárias, todavia, contém predomínio na infância e adolescência, substancialmente pela escassez de uma vida enérgica e uma alimentação desregulada (Oliveira, 2018). Ainda para os autores, os hábitos inapropriados ocasionam doenças 
cardiovasculares, diabetes, hipertensão arterial e como múltiplos outros agravantes a saúde. Diante disso, os autores chamam a atenção e afirmam que é indubitável o valor da AF rotineira, influenciando de maneira eficiente na precaução de enfermidades, na adição da predisposição e nos fatores de socialização.

É oportuno destacar que as atividades agradáveis e adequadas aos progressos de menores de idade, como as danças, jogos, brincadeiras, esportes e caminhadas servem como forma de aproveitar o tempo disponível para realização desses, distanciando-os da marginalidade. $\mathrm{O}$ abarcamento desse grupo em atrações desportivas auxilia na diminuição do índice de violência e delinquência, além dos benefícios alcançados pela aplicação do EF (Oliveira, 2018).

Conforme as Recomendações Globais de Atividade Física (2010), pressupõe-se que 27,5\% dos adultos e $81 \%$ dos adolescentes não finalizam as recomendações da EF, em especial dos aeróbicos. Há uma imprescindibilidade na elevação da precedência e dos investimentos direcionados aos setores-chaves para promoção da AF. Nas duas ultimas décadas, esses dados, não tem revelado nenhum crescimento geral nos níveis globais de aviso e diferenciações de gênero. Além disso, os mesmos mostram, consistentemente, desigualdades na participação por sexo, idade, gestação, deficiência, status socioeconômico e geográfico, ampliando a conveniência de reforçar a renda nessas atividades (Guthold et al., 2018; OMS, 2018; Guthold et al., 2020).

Dessa forma, Sátiro et al. (2021) ao investigar os benefícios da AF em período reduzido e de extenso prazo, deduziram que, é profundamente influenciável as práticas saudáveis em comum a execução constante de EF na vida adulta, com elevado impacto, acerca principalmente da prevenção de massa óssea, alta autoestima e a precaução do câncer de mama (Oliveira, 2018; Sátiro et al., 2021).

Independente do período em que se encontra, a $\mathrm{AF}$ consegue apresentar inúmeras vantagens para o praticador. Esse processo conjuntamente com o ambiente, a nutrição e a genética, auxiliam para que o indivíduo alcance seu potencial de crescimento, desenvolva absolutamente a capacidade motora e além disso, tenha em concordância no resultante um favorável nível de saúde (Iucatan, 2020). É imprescindível que AF esteja aliada conjuntamente a uma alimentação saudável, uma vez que fornece carboidratos, proteínas, lipídios, água, minerais, fibras e vitaminas, essenciais para a conservação da saúde (Guiaretta et al., 2020).

\subsection{Os riscos do sedentarismo}

Um estilo de vida ativo traz proveitos e benefícios para o realizante, e, com a prática regular mantém ou/e melhora a

saúde do homem. É pertinente acrescentar que no adulto a manutenção de suas atividades auxilia na prevenção, tratamento ou/e redução de DCNT. Ademais, a adição da educação física contribui decisivamente para a saúde pública e apresenta também um impacto significativo na redução dos custos do tratamento, incluindo hospitalização (Malta et al., 2021; Freitas, 2019; Nunes et al., 2021). Outrossim, é considerada uma aliada contra outros fatores nocivos à saúde, além de atuar contra a dependência de álcool e tabagismo (Carvalho, 2020).

Oliveira (2018) afirma que seus benefícios são vistos em diferentes aspectos físico, psicológico, social e entre outros. Do ponto de vista da saúde, o EF está relacionado à prevenção e a medida terapêutica de múltiplasenfermidades. Sobre essas perspectivas, diversos pesquisadores acreditam que a atividade física rotineira consegue impactar significativamente na precaução e/ou no controle da hipertensão arterial sistêmica, na diabetes não insulino-dependente, obesidade, asma brônquica, osteoporose, dislipidemia e outras doenças pulmonares (Carvalho, 2020).

Todavia, a saúde não se refere, apenas, a sua forma física, mas portam outros aspectos, como a saúde mental e libido, com atividades que produzem sensação de satisfação e direção. Além do que, manter um hábito ativo é prazeroso, encarrega inúmeras regalias, desde a redução do percentual de gordura corporal até o acrescento da auto-estima, podendo ainda, aprimorar a interação social, de forma positiva para o indivíduo (Bull et al., 2020). 
As vantagens de prosseguir com a $\mathrm{AF}$ durante a pandemia também são relatados, sugerindo um efeito positivo na percepção do estresse, qualidade do sono, ansiedade e depressão. Ao investigar as opiniões de indivíduos com 18 anos ou mais sobre os comportamentos afetados pelo isolamento social durante a pandemia de COVID-19, descobriu-se que as pessoas que se exercitavam relataram níveis reduzidos de cortisol (Daronco et al., 2021).

Ademais, sabendo da sua relevância, os estudos mostram que à medida que as pessoas envelhecem, tornam-se mais sedentárias, principalmente, pelo consumo médio diário de energia ou aos fatores comportamentais e sociais, como melhor comprometimento estudantil e/ou profissional apresentando uma tendência decrescente à custa da redução das AF (Bull et al., 2020).

Daronco et al. (2021) afirmam que mesmo após a recuperação do COVID-19, o paciente ainda precisará manter o hábito de práticas físicas. Os autores argumentam ainda que os pacientes sobreviventes precisam seguir as recomendações da Sociedade Brasileira de Cardiologia e da Sociedade Brasileira de Medicina Exercício e do Esporte, onde devem se submeter á uma avaliação exaustiva de exercícios na região torácica posterior. Esse alerta é especialmente importante para pacientes hospitalizados, pois cerca de $20 \%$ a $30 \%$ possui algum envolvimento cardíaco.

\subsection{Atividade física na infância: vantagens e recomendações.}

Desde criança, é importante a realização da $\mathrm{AF}$, uma vez que as pesquisas apontam que os resultados são positivos, onde incluem a redução dos sintomas de ansiedade, depressão e a proteção de doenças crônico-degenerativas (Bull et al., 2020). Além do mais a prática de exercícios em grupo (futebol ou voleibol) contribui para a socialização e ensina aos joviais, a respeitarem as regras. Perder uma disputa é uma forma de aprender a lidar com contratempos, ensina a persevera e a superar obstáculos. Ganhar é colocar a insígnia de orgulho, felicidade, avanço a auto-estima. Como descrito, crianças ativas desfrutam da oportunidade de tornarem-se adultos ágeis. Ademais, para ocorrer dessa forma, tende-se a realizar, simultaneamente atividade que gerem agrado para o praticante, de forma a apresentar aos alunos as vantagens de se conseguir executar as técnicas e que estas se tornem habituais em suas vidas (Arraz, 2018).

A AF constante e apropriada proporciona o desenvolvimento saudável da estrutura óssea, permitindo que o indivíduo siga sua carreira genética de amplificação longitudinal e transversal. Além disso, exerce uma função essencial sobre o estado físico, mental e psicológico. A escassez ou excesso dessa, afeta o desenvolvimento em estatura e causam deformações ósseas. (Bull et al., 2020; Lima et al., 2019).

Através das pesquisas realizadas entre jovens estudantes, averígua-se que os mesmos correlacionam os resultados favoráveis da execução da AF a tópicos gerais (Arraz, 2018):

1) Execução - com o crescimento pessoal e o reconhecimento social;

2) Benefícios psicológicos - relativos ao humor e ao acréscimo de confiança;

3) Benefícios físicos - relacionados à aparência, desempenho físico e benefícios à saúde;

4) Satisfação - resultante da socialização juntamente a outros jovens;

Ao contrário de alguns mitos populares, o EF seguro não é prejudicial para os menores, essa abordagem também promove outros benefícios favoráveis para a saúde delas, como: (Carvalho, 2020; Bull et al., 2020):

- Promover o crescimento e o desenvolvimento;

- Fortalecer ossos, músculos e articulações;

- Aprimorar a postura e o equilíbrio; 
- Aumentar a sensibilidade à insulina;

- Aperfeiçoar o status de lipídios no sangue;

- Melhoria da auto-estima;

- Aprimoramento da concentração;

- Domine o corpo.

A prática da AF realizada de forma prazerosa e relaxante possui como finalidade fundamental o desenvolvimento de hábito e incentivo, tornando-se relevante para a saúde e as habilidades sociais. Portanto, as entidades profissionais, científicas e a mídia precisam tratar a AF na infância como um problema de saúde pública, divulgando informações sobre sua importância e a necessidade de implementar planos para sua prática direcionada. Essas devem manter-se interessadas e sempre oferecer alternativas para esse meio, sendo sempre consideradas como interesses pessoais, a fim de atingir os objetivos dos alunos e proporcioná-los diversas experiências (Arraz, 2018).

Portanto, se faz necessário prioriza-se a integração e a educação física escolar, para que possam se agregar à sociedade, ao invés de discriminar as pessoas com menos habilidades. Contudo, quando não gerenciadas adequadamente, pode prejudicar a maturidade individual, nessas condições extremas de treinamento, acompanhadas de outros fatores negativos, costumam aparecer em esportes de alto rendimento, como desnutrição e sobrecarga emocional, levando um atraso na maturidade (Iucatan, 2020).

\section{Conclusão}

Esse estudo retrata que a obesidade e o sedentarismo crescem gradativamente e as principais formas de melhorar é uma modificação na rotina e na qualidade de vida da população infantil, que pode ser realizada por meio de transformações nos hábitos e comportamentos físicos e alimentares, no intuito de evitar complicações na fase adulta. Ao observamos as causas e consequências, percebe-se que a atividade física é indicada para qualquer faixa etária em um processo do desenvolvimento motor da criança, a fim também de combater o sedentarismo e tornando-as mais ativas.

Por meio dos artigos analisados, é possível perceber que as crianças que desenvolvem ou executam atividades sedentárias, tais como: jogos eletrônicos, uso do tablet, celulares, assistir televisão, ler livros e o uso de computadores, apresentam em sua maioria uma composição corporal acima do normal para sua idade, quando comparado as crianças que praticam exercícios físicos constante. As crianças que se exercitam tendem a possuir o metabolismo aprimorado e acelerado, desenvolvendo ossos, músculos e tendões mais firmes, sem apresentar riscos de lesões, possuem uma redução na composição da massa corporal, melhorando com isso, o sono e diminuindo o risco de doenças crônicas.

Vale ressaltar que em qualquer fase da vida que o indivíduo estiver é preciso ter cuidado especiais na forma de execução da prática das atividades físicas, se possível essa deve ser sempre feita com o acompanhamento de um profissional habilitado (Educador Físico). Uma vez que pesquisas demostram que essas atividades físicas quando bem realizada apresentam pontos positivos que pode contribuir significativamente no processo de uma vida saudável em prol do bem estar das pessoas. No entanto, ressalta-se que essa prática quando mal orientada, gera traumas físicos, psicológicos e lesões, tornando-se assim, antagonista a sua finalidade que é o desenvolvimento pessoal.

Por fim, tendo em vista o exposto, notificar-se que através dessa pesquisa, garantir uma infância ativa e de qualidade favorece uma saúde adequada a criança no futuro, pois ao ensinar o valor de costumes e hábitos saudáveis durante o seu crescimento, é possível que a mesma desenvolva uma aptidão pela vida saudável o que auxilia no fortalecimento físico e social. 


\section{Referências}

Arraz, F. M. A. (2018). Importância da Atividade Física na Infância. Revista Científica Multidisciplinar Núcleo do Conhecimento. 0192-103, https://www.nucleodoconhecimento.com.br/educacao/atividade-fisica-na-infancia\#_ftn1.

Bull, F. C., Al-Ansari, S. S., \& Biddle, S., Borodulin, K., Buman, M. P., Cardon, G., Carty, C., Chaput, J. P., Chastin, S., Chou, R., Dempsey, P. C., Dipietro, L., Ekelund, U., Firth, J., Friedenreich, C. M., Garcia, L., Gichu, M., Jago, R., Katzmarzyk, P. T., Lambert, E., \& Willumsen, J. F. (2020). Who 2020 guidelines on physical activity and sedentary behaviour. British Jornal Ofsports Medicine, 54(24), 1451-1462. Doi: 10.1136/Bjsports-2020-102955.

Carvalho, R. B. N. (2020). Padrões de comportamentos de risco e de proteção para doenças crônicas não transmissíveis na população adulta e infantil do Brasil. Tese de Doutorado, Faculdade de Saúde Pública, Universidade de São Paulo, São Paulo. https://www.teses.usp.br/teses/disponiveis/6/6138/tde17022021-161513/publico/CarvalhoRBN_DR_O.pdf.

Campos, C. G., Muniz, L. A., Belo, V. S., Romano, M. C. C., \& Lima, M.C. (2019). Conhecimento de adolescentes acerca dos benefícios do exercício físico para a saúde mental. Ciência \& Saúde Coletiva. 24(8), 2951-2958. DOI: 10.1590/1413-81232018248.17982017

Cech, J., \& Martin, S. Tink. (2011). Motor Development. Functional Movement Development Across the Life Span-E-Book. Elsevier Health Sciences, 45-67.

Daronco, L. S. E., Pozzobon, D., Ramos, D. L., Oliveira, J. M. S., \& Berria, J. (2021). Educação Física e Saúde em Tempos de Covid-19. Observatório Socioeconômico da COVID-19. https://www.ufsm.br/app/uploads/sites/820/2021/04/Textos-para-Discussao-27-Educacao-Fisica-e-Saude-em-Tempos-deCOVID.pdf.

Freitas, M. M. (2019). A influência da atividade física no aprendizado com idade escolar. Revista Científica Do Instituto Ideia. 2 ANO 8. https://revistaideario.com/pdf/desm/revista.ideario.15n.02_2019/revista.ideario.n15_02.2019.063_a.influencia.da.atividade.fisica.pdf.

Gallahue, D. L., \& Ozmun, C. J. (2003). Compreendendo o Desenvolvimento Motor: bebês, crianças, adolescentes e adultos. (3a ed.), Phorte Editora.

Giaretta, M. (2020). Alimentação saudável: educando e cuidando da infância na educação infantil. $11 \mathrm{f}$. Artigo de conclusão de curso (Licenciado em Pedagogia). Curso de Pedagogia. Universidade de Passo Fundo, Passo Fundo, RS, 2020. http://repositorio.upf.br/bitstream/riupf/1928/1/PF2020Mariele\%20Giaretta.pdf.

Guthold, R., Stevens, G. A., Riley, L. M., \& Bull, F. C. (2018). Worldwide trends in insufficient physical activity from 2001 to 2016 : a pooled analysis of 358 population-based surveys with 1.9 million participants. The Lancet. Global health, 6(10), e1077-e1086.

Guthold, R., Stevens, G. A., Riley, L. M., Bull, F. C. (2020). Global trends in insufficient physical activity among adolescents: a pooled analysis of 298 population-based surveys with 1.6 million participants. The Lancet. Child \& adolescent health, 4(1), 23-35. 10.1016/S2352-4642(19)30323-2

Iucatan, M. L. D. (2020). A contribuição do treinamento funcional para a diminuição da obesidade infantil. Trabalho de Conclusão de Curso- Monografia, Pontifícia Universidade Católica de Goiás, Goiânia. https://repositorio.pucgoias.edu.br/jspui/bitstream/123456789/572/1/A\%20Contribu icao $\% 20$ do $\% 20$ Treinamento $\% 20$ Funcional $\% 20$ para $\% 20$ a $\% 20$ diminuicao $\% 20 \mathrm{da} \% 20$ obesidade $\% 20 \mathrm{infanti1} \% 20$.pdf.

Lima, J. de S., Martins, J., Marques, A., \& Silva, A. Y. (2019). Associação entre práticas de atividade física e desempenho acadêmico de estudantes chilenos do ensino fundamental e médio. Revista Brasileira de Ciências do Esporte. 41(2):206-214. 10.1016/j.rbce.2018.03.028.

López-gil, J. F., Caetano, C. I., Sentone, R. G., Cavichiolli, F. R. \& Yustelucas, J. L. (2020). Self-reportedlevelofphysicalactivity in schoolchildren Who participate in physicaleducation: a systematic review. Cadernos Brasileiros de Terapia Ocupacional. 28(3), 1020-1036. 10.4322/2526-8910.ctoAR1962.

Malta, D. C., Gomes, C. S., Barros, M. B. A., Lima, M. G., Almeida, W. S., Sá, A. C. M. G., Prates, E. J. S., Machado, I. E., Silva, D. R. P., Werneck, A. O., Damacena, G. N., Júnior, P. R. B. S., Azevedo, L. O., Montilla, D. E. R., \& Szwarcwald, C. L. (2021). Doenças crônicas não transmissíveis e mudanças nos estilos de vida durante a pandemia de COVID-19 no Brasil. Revista Brasileira de Epidemiologia, 24, e210009. 10.1590/1980-549720210009.

Nunes, M. I. L. M., Matos, A. G. M., Lima, L. E. M., \& Neto, J. G. B. (2021). Atividade física como prevenção da obesidade e síndrome metabólica na infância e adolescência: uma revisão integrativa. Multidisciplinary Reviews, 4, e2021009. 10.29327/multi.2021009.

Nunes, T. T. G., Lara, S., Graup, S., Teixeira, L. P. \& Balk, R. S. (2019). Controle postural na infância: efeitos do Método Pilates sobre o equilíbrio. Revista Brasileira de Ciência e Movimento, 27(1):33-41. 10.31501/rbcm.v27i1.7619.

Oliveira, C. (2018). Educação Física Escolar na Infância e Adolescência e sua influência na adoção da Atividade Física na vida adulta. Trabalho de Conclusão de Curso - Instituto Federal Catarinense Campus Avançado Abelardo Luz Especialização Em Educação, Abelardo Luz/Sc, Agosto de 2018. http://abelardoluz.ifc.edu.br/wp-content/uploads/2018/12/TC-Caroline-Oliveira.pdf.

Sátiro, R. D. S. (2021). Benefícios das práticas de atividades físicas em crianças no âmbito escolar. Artigo de conclusão de curso (Licenciado em Educação Física). Curso de Educação Física. Faculdade Ages, Campus Senhor do Bonfim https://repositorio.animaeducacao.com.br/bitstream/ANIMA/17328/1/TCC\%20Renan\%20S\%c3\%a1tiro\%20vers\%c3\%a3o\%20final\%20RUNA.pdf.

Silva, W. V. \& Veneziano, L. S. N. (2021). A importância da educação física na vida de crianças obesas. Revista Ibero-Americana de Humanidades, Ciências e Educação. São Paulo, 7(10), 1-18. 10.51891/rease.v7i10.2845.

Who. (2018). Plano de ação global para atividade física 2018-2030: pessoas mais ativas por um mundo mais saudável. WHO. https://apps.who.int/iris/bitstream/handle/10665/272721/WHO-NMH-PND-18.5-por.pdf.

Xavier, J. (2018). A importância do desenvolvimento motor na primeira infância. Fundação Oswaldo Cruz (Fiocruz). https://portal.fiocruz.br/noticia/importancia-do-desenvolvimento-motor-na-primeira-infancia. 